\title{
comportement des roches au cours de la. rupture : applications à l'interprétation d'essais sur des tubes épais
}

\author{
par \\ P. Berest \\ J. Bergues \\ Nguyen Minh Duc \\ Laboratoire de Mécanique des Solides \\ Ecole Polytechnique
}

\section{Introduction}

On sait depuis longtemps que le dépassement de la limite élastique, dans des excavations souterraines à grande profondeur, ne remet pas nécessairement en cause la stabilité de l'ouvrage. L'apparition d'écaillage ou de fissuration importante aux parois peut être compatible avecune pérennité suffisante de la cavité.

La théorie del'élastoplasticité parfaite est souvent invoquée pour rendre compte de ces observations. Importée de la Mécanique des Métaux ou des Sols, elle donne pour les massifs rocheux des résultats en général trop optimistes: on vérifie facilement que, dans cette théorie, un tunnel profond creusé dans un matériau à cohésion non nulle ne peut pratiquement pas être instable.

Les raisons de cet optimisme sont illustrées par les figures 1 et 2. Le schéma élastoplastique parfait est représenté à la figure 1. Le comportement réel des roches est tout autre: si on pilote en déplacement un essai de compression simple. l'allure de la courbe obtenue dansle plan $\left(\frac{F}{\delta}, \frac{\Delta l}{l}\right)$ est celle donnée par la figure 2; on observe qu'il y a radoucissement. Si on se place dans le diagramme de Mohr, la plasticité parfaite implique que, passé le point $R$, le cercle de Mohr reste constant (fig. 3.a) si on poursuit le déplacement; dans le cas du radoucissement, il devient plus petit (fig. 3.b).

Plus généralement, dans le cas d'un chargement plus complexe, au moins pour des pressions moyennes pas trop importantes, l'évolution ne se fait pas dans la réalité en suivant la courbe intrinsèque, mais en se déplaçant "à l'intérieur" de la courbe intrinsèque (fig. 4).

On peut en tirer les conclusions suivantes :

La courbe intrinsèque d'une roche représente les valeurs limites des contraintes avant la rupture pour une roche initialement non contrainte et chargée d'une manière monotone; elle ne donne pas de renseignements sur l'évolution de matériau lorsqu'il est placé à la limite de rupture.

II faut remarquer que cette affirmation remet en cause, comme trop optimistes, les calculs de structure fondés sur l'utilisation mathématique du tracé de la courbe intrinsèque. Notons au passage que la méthode originale mise au point au Laboratoire de Mécanique des Roches de l'Ecole des Mines de Paris, J. Fine et al (1979), qui consiste à bloquer le déplacement longitudinal et à piloter les contraintes axiale et transversale, doit être considérée, suivant les prin-



Fig. 1 Schéma élastoplastique parfait



Fig. 2 Comportement d'une roche en compression simple

cipes énoncés ici, non pas comme une recherche de courbe intrinsèque mais comme la détermination de l'enveloppe des cercles de Mohr dans un trajet en contraintes qui dépasse la limite élastique. 


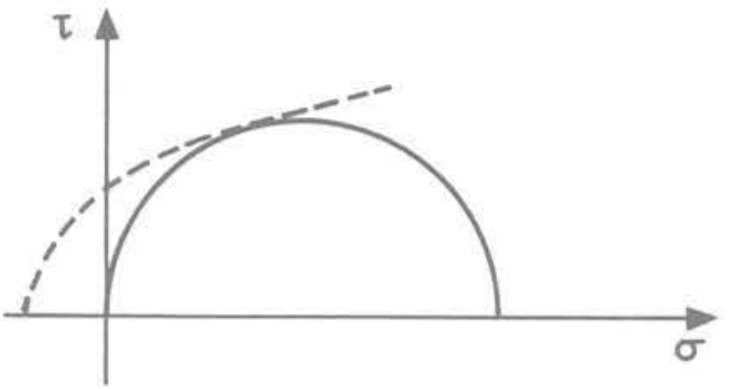

Fig. 3a Elastoplasticité parfaite

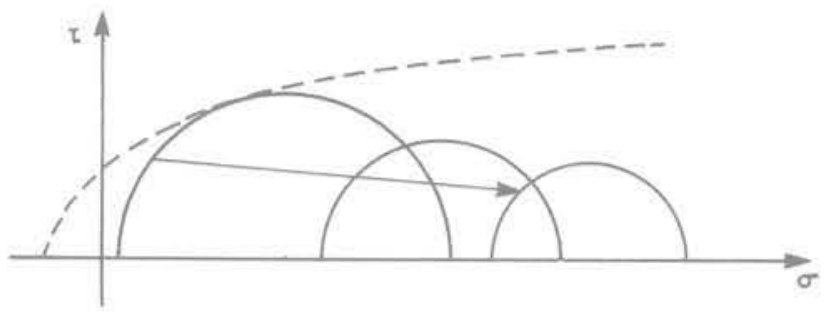

Fig. 4 Trajet de charge

\section{Problèmes posés par une représentation macroscopique du comportement après rupture}

On sait que les roches se distinguent d'autres matériaux par l'existence, même à l'état naturel non contraint, d'un ensemble de discontinuités à des échelles très diverses. Le jeu de ces discontinuités (ouverture et développement des fissures) intervient dans le comportement au moins après la phase dite "linéaire" de l'essai en compression simple; il est manifestement prépondérant au voisinage et au-delà du maximum de la force (Fig. 2).

Ceci implique deux conséquences majeures qui sont de graves obstacles à une formalisation plus satisfaisante que l'élastoplasticité parfaite.

2.1 La courbe force-déplacement d'une éprouvette ne traduit de façon correcte le comportement intrinsèque de la roche que tant que les dimensions des discontinuités restent, par rapport à celles de l'éprouvette, suffisamment petites pour que les hypothèses de contraintes axiales et de déformations homogènes conservent un sens. Lorsque les discontinuités interagissent suffisamment pour qu'apparaissent des surfaces de glissement macroscopiques, le comportement global n'est plus la somme des comportements supposés identiques de chaque partie de l'échantillon; le rôle des fissures macroscopiques devient prépondérant. Un exemple élémentaire est le cas de la compression simple, lorsqu'apparaît un plan de glissement incliné sur l'horizontale (fig. 5).

Dans l'expérience sur des cylindres creux qui fait l'objet de la suite de cet article, ce phénomène se manifeste par l'apparition d'une surface de rupture qui rejoint les parois intérieure et extérieure (fig. 6)

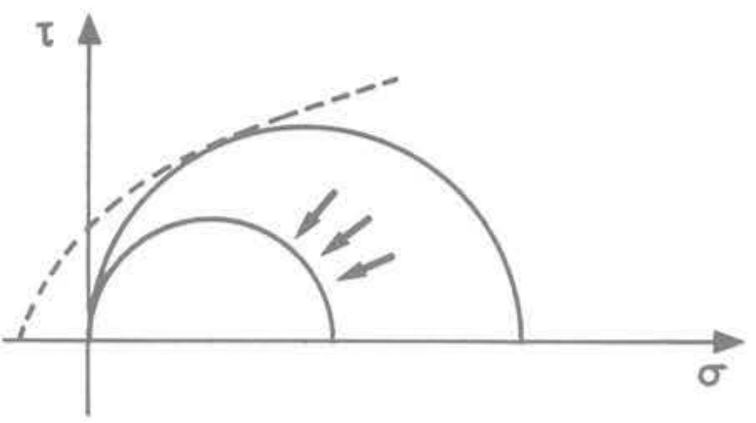

Fig. 3b Radoucissement

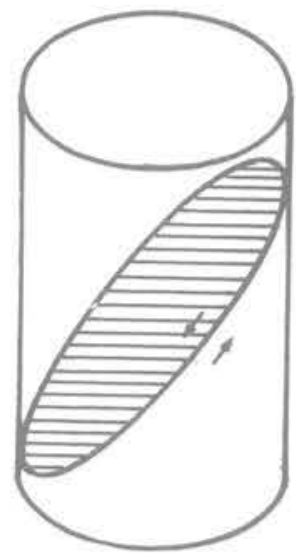

Fig. 5 Plan de glissement en compression

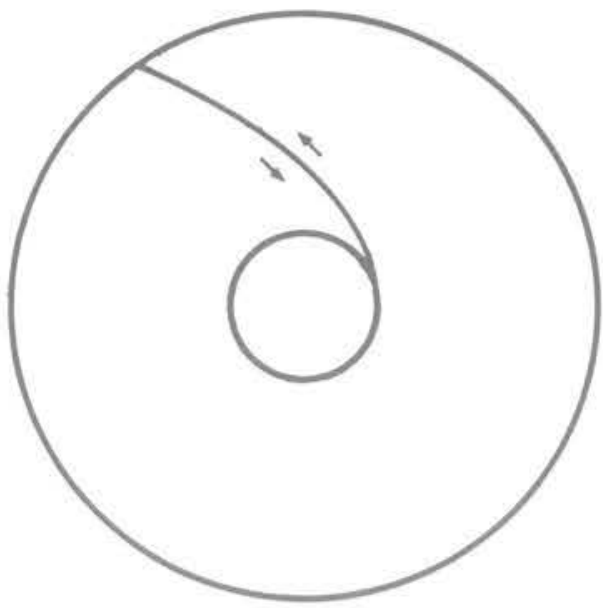

Fig. 6 Surface de glissement dans l'écrasement radial d'un tube

2.2 Le comportement après la rupture est très influencé par la contrainte moyenne; les essais sur éprouvettes en témoignent, par une augmentation notable avec la contrainte moyenne de la pente de la courbe effortdéplacement après la rupture. On peut également rapprocher ces faits de la remarque de E. Tincelin, (1979), sur l'efficacité, pour la tenue d'un ouvrage, d'un confinement même faible (il faut toutefois distinguer dans ce cas roche au voisinage de la rupture encore cohérente et roche broyée). Pour l'instant, les modèles proposés tiennent mal compte de cette influence; on le verra dans l'interprétation des essais sur cylindre creux. 

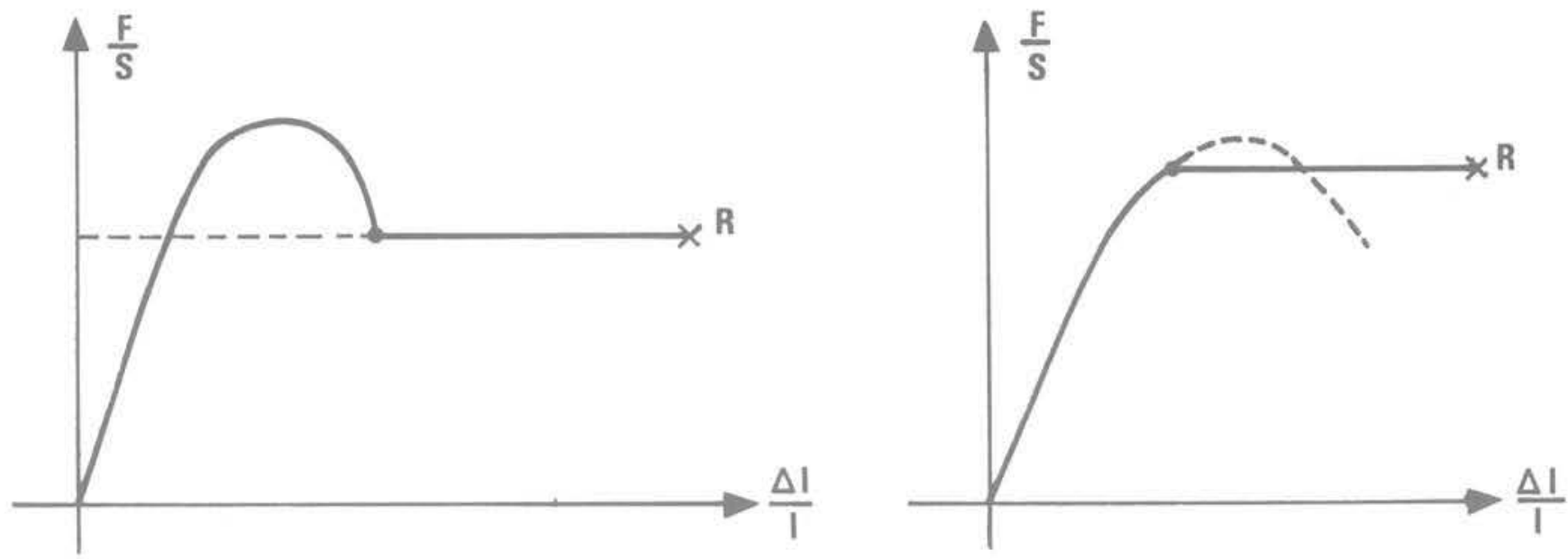

Fig. 7 Fluage au voisinage du maximum

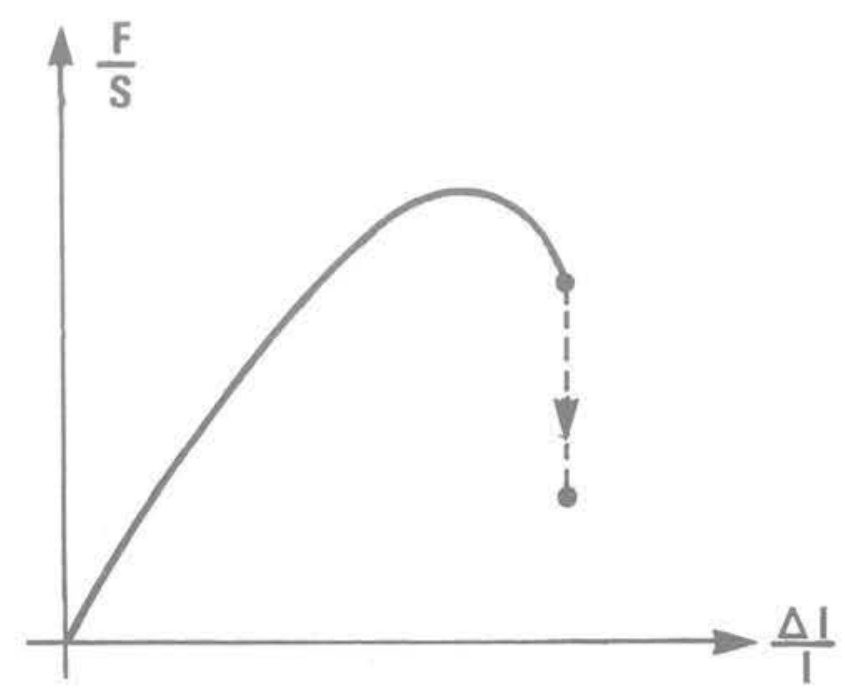

Fig. 8 Relaxation après le maximum

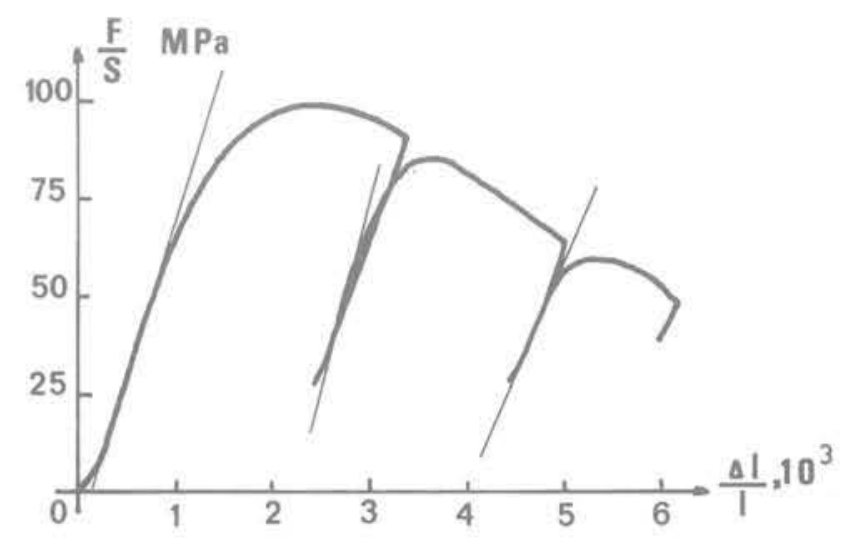

Fig. 9 Charges et décharges sur un marbre de Carrare
2.3 Linfluence de la vitesse de chargement est sans doute le phénoméne le plus caractéristique, à tel point qu'on peut formuler la règle suivante : toutes les roches ont un comportement visqueux au voisinage de la rupture. Rappelons quelques-uns des faits qui mettent cette propriété en évidence :

a Une expérience de fluage à partir d'un point situé dans la partie après le maximum conduit toujours à la rupture. Il en est de même pour des points situés avant le maximum mais proches de celui-ci (fig, 7).

b Les expériences de relaxation, à partir d'un point situé dans la partie après le maximum, sont toujours marquées par une chute de la charge sollicitante (fig. 8).

c Les expériences de décharge suivie d'une recharge, dans la partie après le maximum, montrent un non recouvrement qui atteste d'un comportement différé (fig. 9).
Les mécanismes intimes qui expliquent ces propriétés ne sont pas connus; ils tiennent sans doute au mode de développement de la fissuration, et peut-être au rôle qu'y jouent les fluides (l'eau en particulier). Ils expliquent peut-être, au moins pour une part, pourquoi la convergence dans les ouvrages souterrains est souvent différée, alors même que le massif est composé de roches auxquelles on n'a pas reconnu de comportement visqueux au sens ordinaire du terme (c'est-à-dire avant la rupture).

Une hypothèse simplificatrice, qui sera faite par la suite, consiste à supposer qu'il existe des relations de comportement valables pour une vitesse de déformation nulle; pour des chargements simples, monotones et bornés, on peut alors considérer qu'après un temps suffisamment long, la structure vérifie ces relations "à vitesse nulle". Notons qu'elles seront dans tous les cas moins optimistes que les relations déterminées par des expériences classiques. 

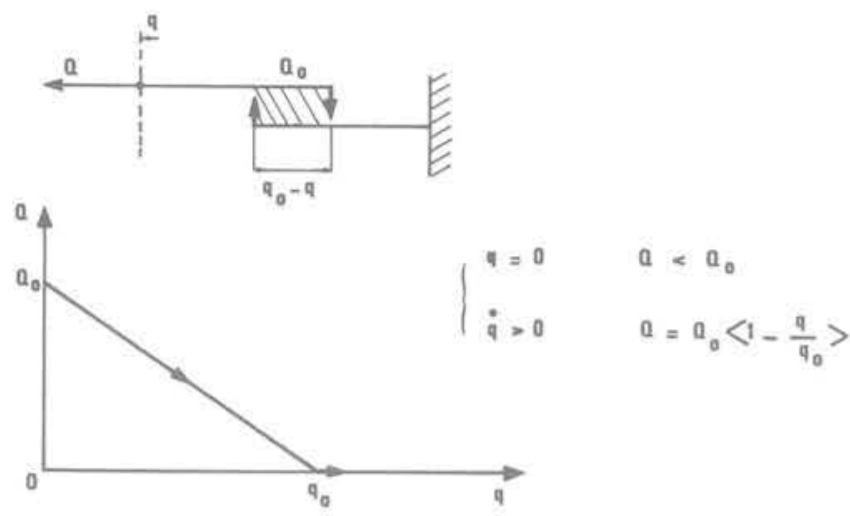

Fig. 10 Patin radoucissant $\left(Q_{0}, q_{0}\right)$

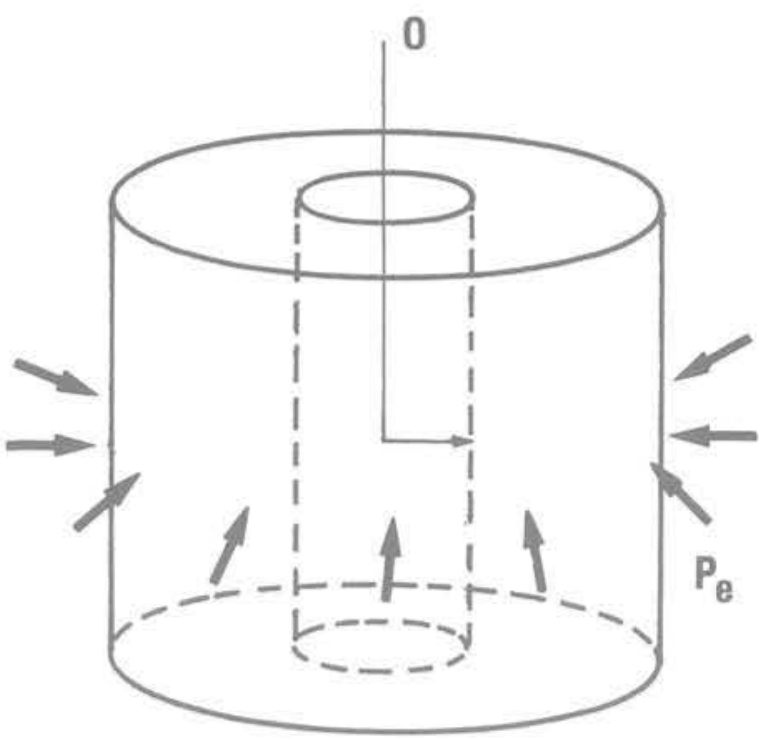

Fig. 12 Ecrasement radial d'un tube

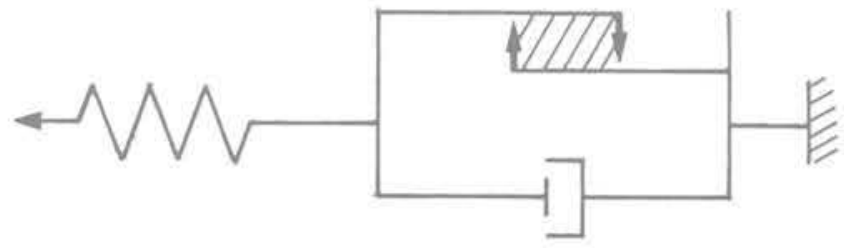

Fig. 11a Modèle élasto-viscoplastique radoucissant

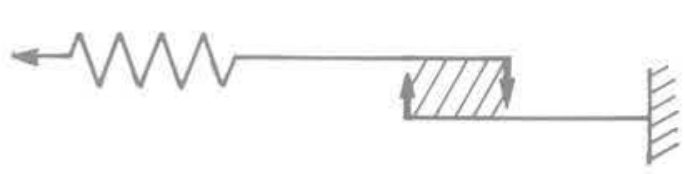

Fig. $11 \mathrm{~b}$ Modèle élastoplastique radoucissant

\section{Modèle proposé pour rendre compte des phénomènes}

Nous avons proposé un modèle de comportement radoucissant pour lequel différents calculs ont été effectués (critère de Tresca et critère de Coulomb avec radoucissement. géométries sphérique et cylindrique, petits et grands déplacements) dont on trouvera un résumé dans Berest et Nguyen Minh Duc (1979). Nous renvoyons aux Annexes pour la description et les résultats essentiels, qui serviront de guide à l'interprétation des expériences sur cylindres creux.

Afin de disposer d'une interprétation rhéologique simple de ce modèle, nous proposons un élément appelé "patin radoucissant", représenté sur la figure 10 (le symbole $\langle>$ signifie "valeur positive de...") : la force nécessaire pour déplacer le patin est proportionnelle à la distance comprise entre les deux flèches, elle diminue avec le déplacement. Le comportement après la rupture est donc décrit qualitativement par le modèle de la figure 11.a; on peut éventuellement rendre compte d'une cohésion résiduelle $Q_{1}$, en groupant en parallèle le patin radoucissant $\left(Q_{0}-Q_{1}\right)$ et un patin ordinaire $Q_{1}\left(Q_{1}<Q_{0}\right)$. Les remarques précédentes sur la viscosité permettent de s'affranchir de l'amortisseur en considérant le comportement après un temps théoriquement infini (fig. 11.b).

\section{Description des essais sur tubes épais}

Une vérification expérimentale des lois précédentes a été tentée sur des tubes épais de roche. Un chargement particulièrement facile à réaliser consiste à imposer une pression croissante sur la paroi extérieure du tube, en laissant l'intérieur en liaison avec la pression atmosphérique (fig. 12).

Afin de mettre en évidence une valeur maximale éventuelle de la charge prédite par le calcul, on a asservi la pression extérieure pour réaliser une vitesse de déformation tangentielle constante sur la paroi intérieure (en supposant une symétrie cyclique $\dot{\epsilon}_{\theta}\left(r_{i}\right)=\frac{u_{i}}{r_{i}}$ est indépendant du point choisi).

La conduite de l'expérimentation devait satisfaire aux préoccupations suivantes.

a Pour faciliter l'interprétation des résultats, il était souhaitable de se rapprocher, le plus possible, d'un état de déformation plane $\left(\epsilon_{z}=0\right)$. Pour cela, la déformation axiale du tube a été empêchée par deux flasques reliées par une barre d'acier centrale.

b L'utilisation des modèles exige que l'influence de la viscosité soit éliminée, on a imposé une vitesse de déformation $\hat{\epsilon}_{\theta}\left(r_{1}\right)=$ cte $=10^{-5} / \mathrm{s}$, égale à la vitesse de "déformation" longitudinale $\left(\frac{\Delta l}{l}\right)$ des éprouvettes utilisées pour la détermination des caractéristiques mécaniques du matériau. 
c II était intéressant, pour un matériau donné, de faire varier le rapport des rayons intérieur et extérił $\left(\frac{u_{i}}{r}\right)^{s}$ éprouvettes avaient donc les dimensions suive... diamètre extérieur : $100 \mathrm{~mm}$

hauteur

$125 \mathrm{~mm}$

et les rayons intérieurs :
$r_{i}=32 \mathrm{~mm}$
soit
$\rho=3.13$
$r=40 \mathrm{~mm}$
soit
$\rho=2.50$
$r_{1}=52 \mathrm{~mm}$
$\rho=1.92$

en notant $\rho$ le rapport des rayons extérieur et intérieur.

d Le matériau choisi devait avoir, en compression simple, un comportement radoucissant marqué. On a choisi une craie sèche, dont les caractéristiques mécaniques étaient les suivantes :

résistance à la compression simple : $R_{c}=8 \mathrm{MPa}$ module élastique : $\mathrm{E}=8900 \mathrm{Mpa}$ coefficient de Poisson

$$
\nu=0.30
$$

La courbe intrinsèque relative à la rupture est, jusqu'à 10 $\mathrm{MPa}$ de pression latérale, assimilable á une droite de Coulomb avec :

angle de frottement : $\varphi=34^{\circ}$

cohésion $\quad \mathrm{C}=2.1 \mathrm{MPa}$

On a également utilisé un matériau beaucoup plus résistant. le calcaire d'Euville, qui présente les caractéristiques suivantes :
$R_{c}=$
$35 \mathrm{MPa}$
$\mathrm{E}=$
$38000 \mathrm{MPa}$
$y=$

Les courbes dans le plan ( $\frac{\Delta l}{l}, \frac{F}{S}$ ) pour une compression simple sont présentées sur les figures 13 et 14 .

\section{Résultats des essais}

La figure 15 présente dans le plan $\left(\frac{U_{i}}{r}, p_{e}\right)$ les résultats des expériences effectuées sur la craie sèche pour plusieurs valeurs des rayons intérieurs.

Notons :

$P_{E}$ : valeur de la pression pour laquelle la courbe $P_{e}=P_{e}\left(\frac{U_{i}}{r}\right)$ cesse d'être linéaire;

$P_{R}$ : valeur de la pression maximale (éventuellement).

On a porté en pointillés pour chacun des cas la courbe qui résulte de l'application du modèle élastoplastique parfait, en prenant les valeurs des paramètres précisées plus haut (le calcul correspondant est précisé en Annexe).

On peut tirer de l'analyse de ces courbes les observations suivantes :

a à charge donnée, les déformations réelles sont, au-delà du point $P_{E}$, beaucoup plus importantes que ne le laisse prévoir le modèle élastoplastique parfait;

b on observe une valeur maximale de la pression extérieure particulièrement nette lorsque le rapport $p$ est petit:

c cette valeur maximale correspond à un déplacement $u$ très nettement distinct du déplacement uÉcorrespondant à la limite élastique;

d on observe un écaillage qui est assez uniformément réparti pour les petites déformations, (ce qui permet de ne pas rejeter l'hypothèse de symétrie cylindrique), et qui affecte l'intérieur du tube. La photo de la figure 16 montre l'aspect de cet écaillage qui apparait avant que le maximum $\mathrm{P}_{\mathrm{A}}$ ne soit atteint:

e pour des déformations importantes, on note une localisation des déformations et de l'écaillage, avec apparition de lignes de glissement qui entraînent une perte de symétrie (figure 17).

L'ensemble des quatre premières observations constitue une confirmation qualitative de P'insuffisance du modèle élastoplastique parfait; a contrario elles confirment les résultats des calculs qui utilisent un modèle d'écrouissage négatif. Il était intéressant de vérifier si un ajustement quan-

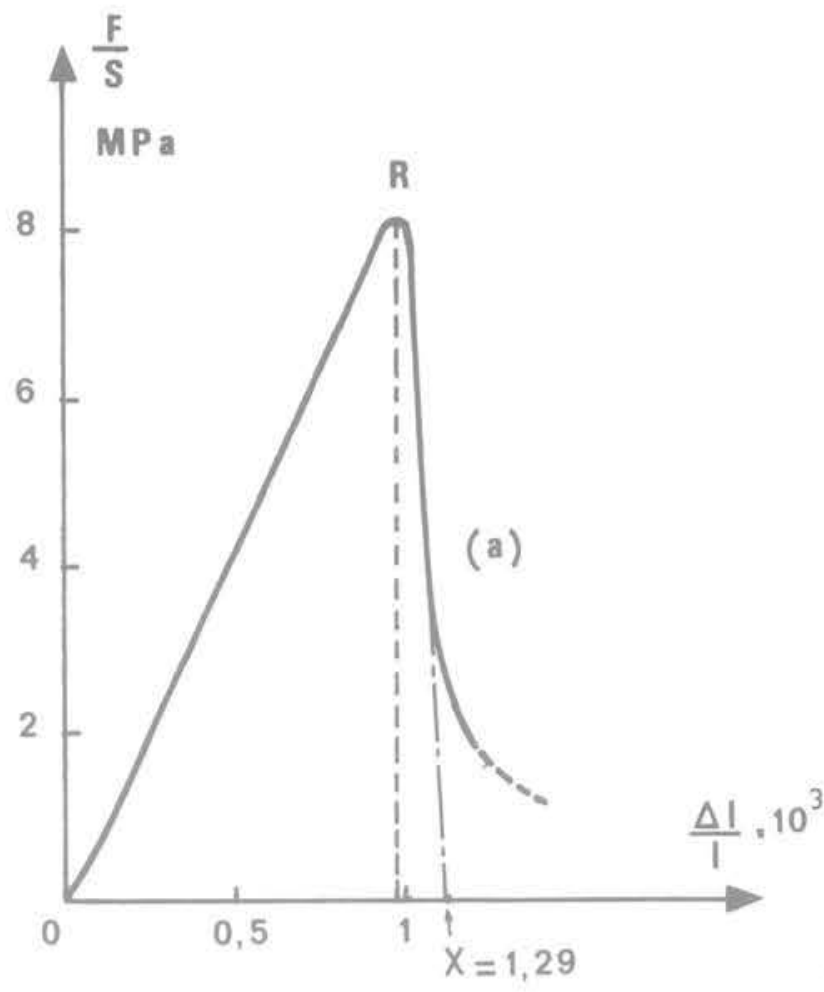

Fig. 13 Essai de compression simple sur une craie

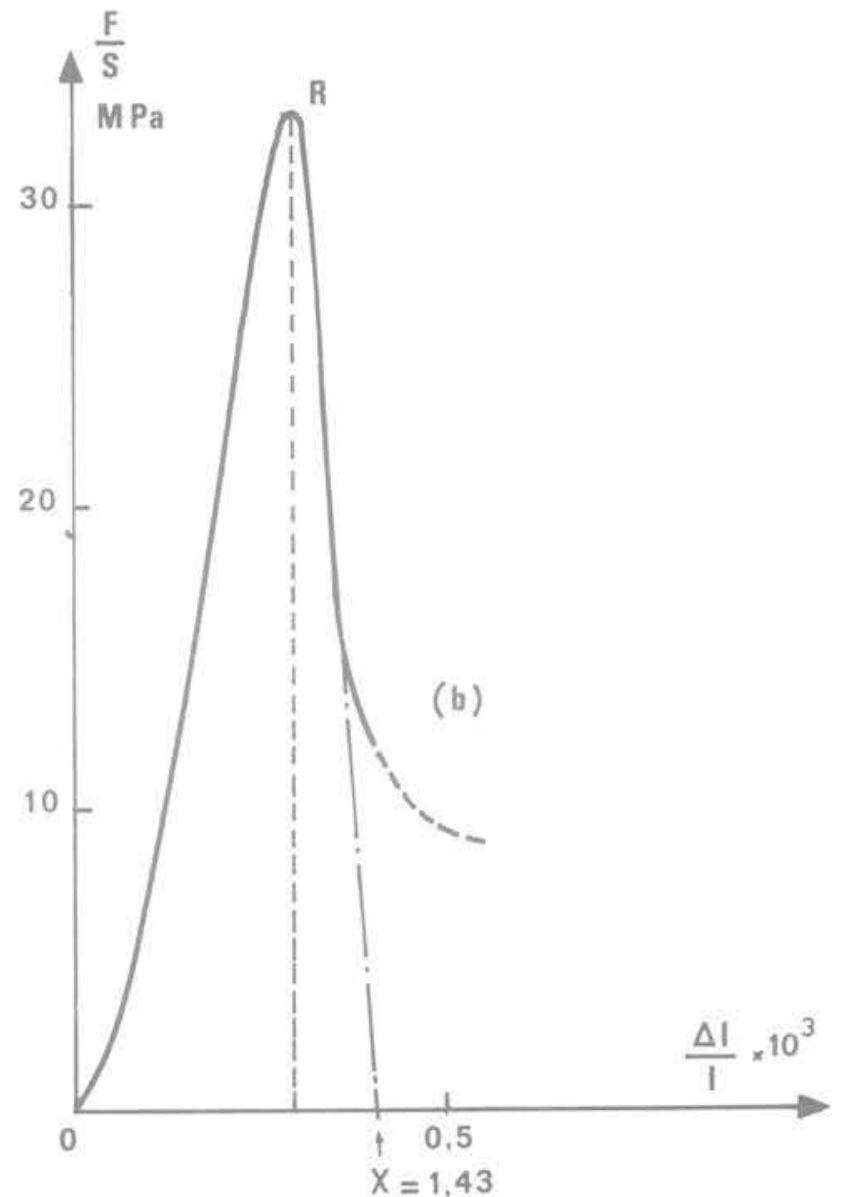

Fig. 14 Essai de compression simple sur un calcaire 


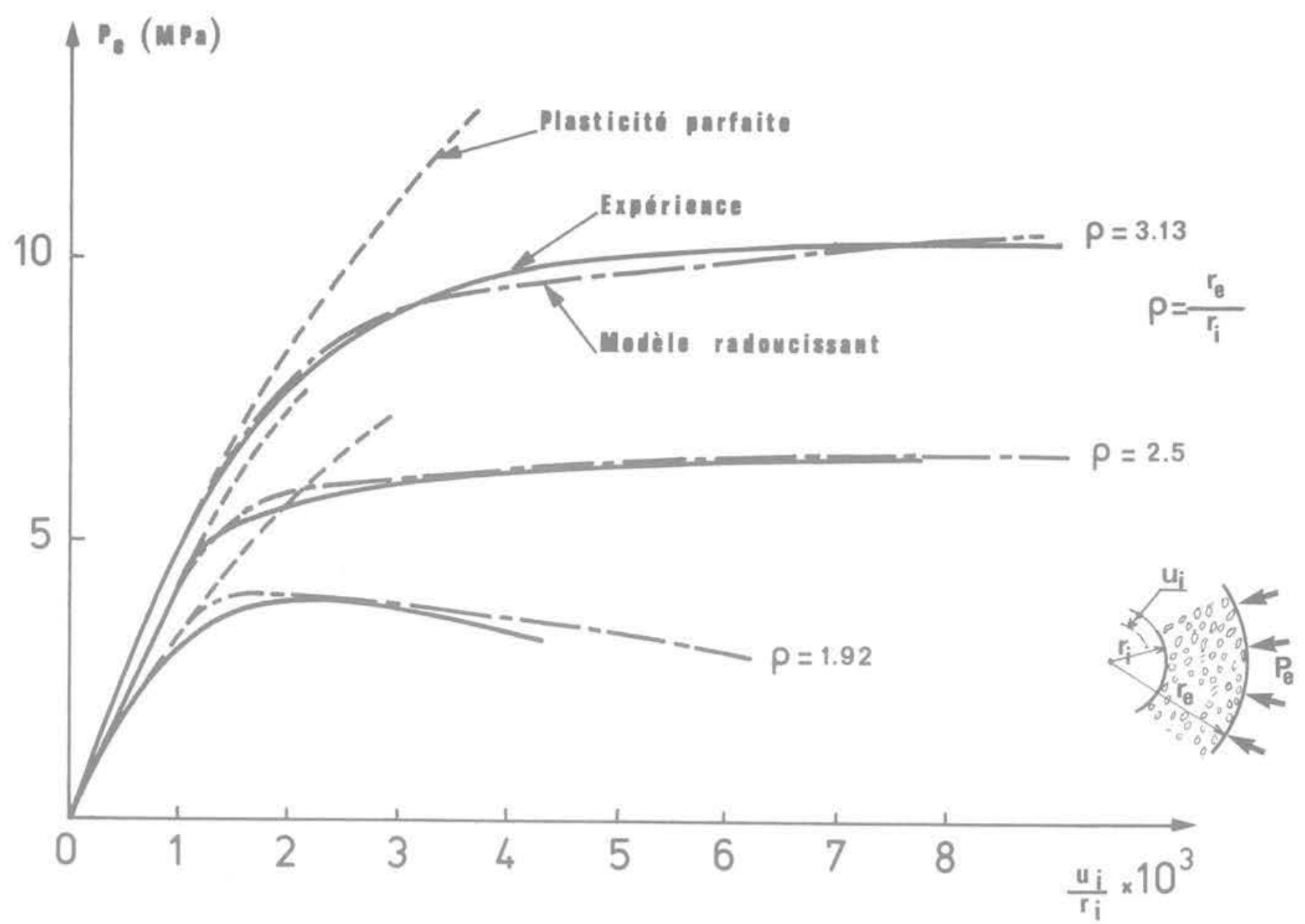

Fig. 15 Comparaison des résultats expérimentaux avec des modèles théoriques

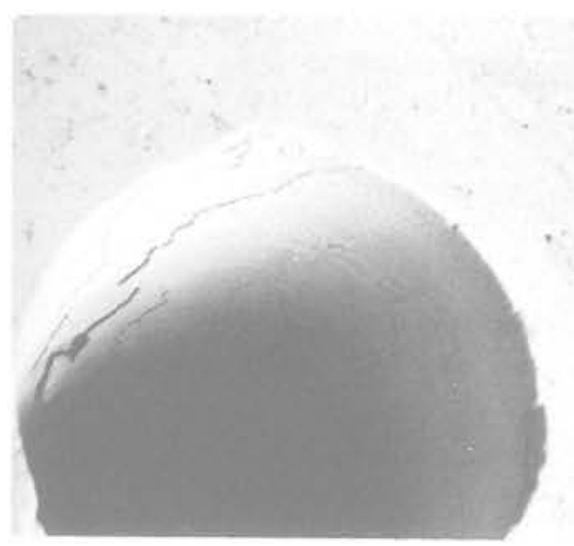

Fig. 16 Ecaillage à la paroi interne de l'éprouvette

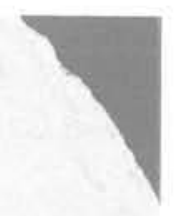

titatif était possible. Cet ajustement est effectué sur la même figure 15, Deux faits sont à noter:

- il est possible de trouver un bon ajustement, comme le montre la figure;

- mais l'ajustement oblige à choisir un $x$ différent pour chaque diamètre, et de toute manière différent de celui de la compression simple.

( $X$ : paramètre proportionnel à la pente après le pic dans le plan effort-déplacement, voir figure 18).



Fig. 17 Ligne de glissement macroscopique sur l'éprouvette

$$
\begin{array}{clr}
\text { Compression simple : } & x=1,29 & \text { (mesuré) } \\
\rho=1,92 & x=1,7 & \text { (ajusté) } \\
\rho=2,5 & x=2,5 & \text { (ajusté) } \\
\rho=3,13 & x=5, & \text { (ajusté) }
\end{array}
$$

La cohésion résiduelle peut être choisie identique dans les quatre cas ( $\left.\mathrm{C}_{0}=0,4 \mathrm{MPa}\right)$. 11 est vrai que son poids dans les résultats numériques est assez faible.

Ces faits montrent que le modèle présenté est trop simple : il ne rend pas compte de la variation de la pente de la partie descendante de la courbe effort-déformation avec l'état de contraintes que mettent bien en évidence les essais triaxiaux. 


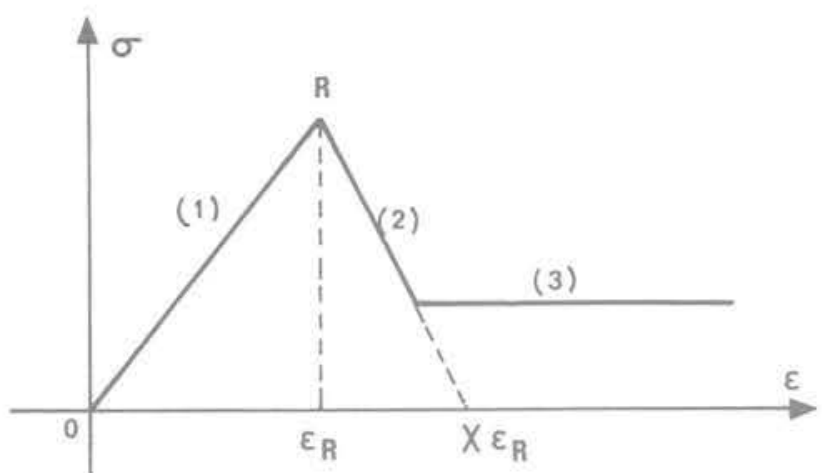

Fig. 18 Schématisation du modèle radoucissant
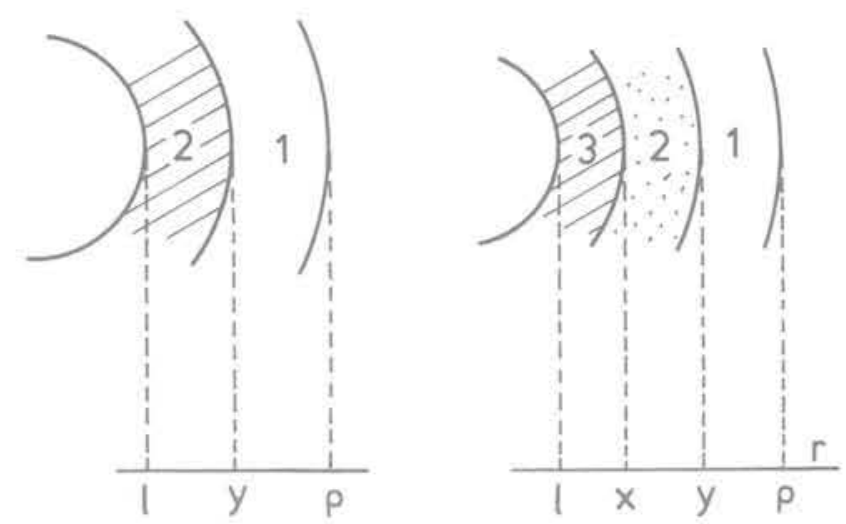

Fig. 19 Développement des zones plastiques

- dans l'invariance du paramétre $\mathrm{K}_{\mathrm{p}}$ (soit de l'angle de frottement $\varphi$ ) et des paramètres élastiques.

Son avantage principal réside dans la possibilité d'obtenir une solution simple dont une discussion complète est possible.

\section{Annexe II}

On rappelle ici certains résultats utiles dont la démonstration complète est dans Nguyen Minh Duc, P. Berest, (1977) et 1979) et P. Berest, Nguyen Minh Duc, M. Panet (1978). On considère un cylindre de révolution creux, de rayon intérieur $r_{i}$, et de rayon extérieur $r_{e_{1}}$ initialement àl'état naturel. Par la suite, on laisse la paroi intérieure libre de contraintes; la paroi extérieure est soumise à une pression uniforme $p_{\mathrm{e}}$ variable, de façon à obtenir une diminution du rayon intérieur (contraction), le tube étant maintenu dans un état de déformation plane $\left(\epsilon_{x}=0\right)$.

Le matériau constitutif obéit à la loi de comportement exposée en Annexe I. On montre que sous le chargement cidessus la déformation plastique se fait uniquement en régime de face (trois contraintes principales distinctes). Pour simplifier l'écriture des formules, on note :

Nous rappelons brièvement le modéle expose par ailleurs Nguyen Minh Duc, M. Panet (1978).

La phase élastique (avant la rupture) est déterminée par les deux coefficients $E$ (module d'Young) et $\nu$ (coefficient de Poisson).

En notant $L$ le tenseur d'èlasticité, on a ensuite :

(1.a.1) $\dot{\epsilon}=L \dot{\sigma}+\dot{\lambda} \frac{\delta \mathrm{g}}{\delta \sigma}$

Avec $\lambda$ multiplicateur plastique et $\mathrm{g}$, potentiel plastique :

(1.a.2) $\quad \mathrm{g}\left(\sigma_{1}, \sigma_{3}\right) \equiv \beta \sigma_{1}-\sigma_{3}$

les contraintes principales étant $\sigma_{1}>\sigma_{2}>\sigma_{3}$; elles vérifient par ailleurs le critère variable de plasticité :

(1.a.3) $\quad f\left(\sigma_{1}, \sigma_{3}\right) \equiv K_{p} \sigma_{1}-\sigma_{3}-\left(K_{p}-1\right)\left[C_{1}-\left(C_{1}-C_{0}\right) \frac{\lambda}{\lambda_{1}}\right]$ Plutôt que le paramètre $\lambda_{0}$, assez lourd à manier, on utilise le parametre $x$ dont l'interprétation est donnée par la figure 18.

$C_{\text {i }}$ est la cohésion initiale

$\mathrm{C}_{0}$ est la cohésion résiduelle

$\mathrm{K}_{\mathrm{p}}, \beta, \lambda_{0}$ sont des constantes

$K_{p}$ : coefficient de butée

$\beta \in\left[1, K_{\mathrm{p}}\right]$ permet de rendre compte d'une dilatance.

On remarquera que ce modèle s'inscrit dans le cadre théorique précisé par Bui et Nguyen Quoc Son (1974). Ses principaux défauts résident :

- dans la liaison finéaire entre le critère f et la variabie cachée $\lambda$ : dans la réalité le paramètre $x$ dépend de l'état de contrainte:

$$
\begin{aligned}
\rho=\frac{r_{e}}{r_{i}} \quad r & =\frac{R}{r_{i}} \text { où } R=\begin{array}{c}
\text { distance à fraxe d'un point } \\
\text { courant; }
\end{array} \\
y & =\frac{Y}{r_{c}} \text { où } Y=\text { rayon de la zone plastique. }
\end{aligned}
$$

On note aussi $\alpha_{1}$ et $\alpha_{2}$ les racines réelles $\left(\alpha_{1}<\alpha_{2}\right)$ de l'équation

(2.a.1) $\alpha^{2}+\left\{2-\frac{\left(K_{p}-\beta\right) x}{x-1+v^{2}}\right\} \alpha-\frac{(1+\beta)\left(K_{p}-1\right)}{x-1+v^{2}} X=0$

Les formules ci-dessous donnent l'expression de la pression $\mathrm{p}_{e}$ et de la déformation tangentielle $\epsilon_{\theta}(1)=\frac{u_{i}}{r_{i}}$ à la paroi du tube, en fonction du rayon de la zone plastique $\mathrm{r}=\mathrm{y}$.

On distingue, (voir figure 19):

a La zone 2 avec radoucissement apparait seule : on note $\mathrm{H}=\mathrm{C} \operatorname{cotg} \varphi$

$$
\begin{aligned}
\mathrm{P}_{\mathrm{e}}+\mathrm{H}= & \frac{H\left(\alpha_{2}-\alpha_{1}\right)}{\left(\alpha_{2}-\left(\mathrm{K}_{p}-1\right)\right) \mathrm{y}^{-\alpha_{1}}-\left(\alpha_{1}-\left(\mathrm{K}_{p}-1\right)\right) \mathrm{y}^{-\alpha_{5}}} . \\
& \frac{\left(K_{p}+1\right) p^{2}-\left(K_{p}-1\right) y^{2}}{2 p^{2}}
\end{aligned}
$$


(2.a.2)

$\frac{u_{i}}{r_{i}}=H \frac{1-v^{2}}{E}\left(K_{p}-1+\frac{\left(K_{p}^{2}-1\right) y^{\alpha_{2}-\alpha_{1}}-1}{\left(\alpha_{2}-\left(K_{p}-1\right)\right) y^{\alpha_{2}-\alpha_{1}}-\left(\alpha_{1}-\left(K_{p}-1\right)\right)}\right)$

b le développement d'une zone plastique parfaite 3 cohésion résiduelle $\mathrm{C}_{0}$ à la suite de la zone I :

Si l'écoulement libre $(y=\rho)$ n'arrive pas auparavant, une zone parfaitement plastique 3 va se développer à partir de la paroi de la cavité $\lambda \geqslant \lambda_{0}$.

La condition $\lambda=\lambda_{0}$ détermine la frontière $r=x$ de lazone 3 en fonction de $y$ : (on note $\mathrm{H}_{0}=\mathrm{C}_{0} \operatorname{cotg} \varphi$ ):

(2.a.3)

$$
\begin{aligned}
1= & \left(1-v^{2}\right) \frac{K_{p}+1}{X-1+v^{2}} \cdot \frac{H-H_{0}\left(1-x^{K p-1}\right)}{H-H_{0}} . \\
& \frac{\frac{y^{\alpha-\alpha_{2}}}{X}-1}{\left(\alpha_{2}-\left(K_{p}-1\right)\right)\left(\frac{Y}{X}\right)^{\alpha_{2}=\alpha_{1}}-\left(\alpha_{1}-\left(K_{p}-1\right)\right)}
\end{aligned}
$$

(avec $\mathrm{x}=1$ dans la formule ci-dessus, on obtient l'instant $y=y_{t}$ d'apparition de la zone (I)

$P_{e}$ et $\epsilon_{\theta}(1)$ ont alors les expressions suivantes :

$$
P_{\mathrm{e}}+\mathrm{H}=\left(H-H_{0}\left(1-\mathrm{K}_{\mathrm{p}}-1\right)\right) \frac{\alpha_{2}-\alpha_{1}}{\left(\alpha_{2}-\left(K_{p}-1\right)\right)\left(\frac{\mathrm{y}}{\mathrm{x}}\right)^{-\alpha_{1}}-\left(\alpha_{1}-\left(K_{p}-1\right)\right)\left(\frac{\mathrm{y}}{\mathrm{x}}\right)^{-\alpha_{2}}}
$$

$\frac{u_{i}}{r_{i}}=f_{\theta}(1)=\frac{1-V^{2}}{E}\left(H_{0}\left(K_{p}-1\right)+\frac{H_{0}\left(K^{2} p-1\right)}{\beta+K_{p}}\left(x^{\left.\beta+K_{p}-1\right)}+\right.\right.$

$$
\left.\frac{x}{K_{D}-1}\left(H-H_{0}\right) x^{\beta+1}\right)
$$

C Cas du matériau parfaitement plastique :

Avec $X-\infty$, on retrouve le comportement parfaitement plastique. D'après 2.a.1., on a alors :

$$
\alpha_{1} \rightarrow-(\beta+1) \quad \alpha_{2}-K_{p}-1
$$

La formule 2.a.2 donne alors, par passage à la limite :

$P_{e}+H=\frac{H y^{K p-1}}{2 p^{2}}\left(y^{2}\left(1-K_{p}\right)+p^{2}\left(1+K_{p}\right)\right)$

(2.a.5)

$\frac{u_{i}}{r_{i}}=\frac{H\left(1-v^{2}\right)}{E}\left(\frac{K_{p}^{2}-1}{\beta+K_{p}}\left(y^{\beta+K_{p}}-1\right)+K_{p}-1\right)$
On remarquera par $(2, a .3)$ que $X \rightarrow \infty$ implique que la zone 3 n'apparaît jamais si $\mathrm{H}_{0}<\mathrm{H}$. Les formules ci-dessus s'obtiennent également par (2.a.4) avec $\mathrm{H}_{0} \rightarrow \mathrm{H}$.

Remarque : le calcul des formules 2.a.2. et 2.a.3., 2.a.4 est en tout point analogue à celui de P. Berest et Nguyen Minh Duc (1977) effectué dans le cas de la symétrie sphérique. On peut montrer ici aussi que $P_{e}$ fonction de $\frac{u_{1}}{r}$ (1) admet toujours un minimum avant l'écoulement libre. Dans le cas où la pression intérieure n'est pas nulle, il peut apparaître, en déformation plane, un régime d'arête. La discussion de ce cas est développée dans Berest et Nguyen Minh Duc (1979).

\section{Références Bibliographiques}

BEREST P., NGUYEN M. D. PANET M., (1978) - Contribution á l'étude de la stabilité d'une cavitè souterraine dans un milieu avec radoucissement, Revue Française de Géotechnique, $n^{\circ} 4$, juin 1978.

BUI H.D., NGUYEN Q.S., (1974) - Sur les matériaux élastoplastiques à écrouissage positif ou négatif, Journal de Mécanique, vol. 13, n², juin 1974, pp. 321-342.

EGGER P., (1973) - Einfluss des Postfailure Verhaltens von Fels auf den Tunnelausbau unter besonderer Berücksichtigung des an Kerausbau, Veröff Instit. F. Boden und Felmech. Univ. Karlsruhe H. 57, 1973.

FINE J., TIJANI S.M., VOUILLE G., BOUCLY P., (1979) Détermination expérimentale de quelques paramètres viscoplastiques des roches, C.R. $4^{\circ}$ Congrès de la SIMR, Montreux, septembre 1979, vol. 1, pp. 139-143.

NGUYEN M. D., BEREST P., (1977) - Contraction d'une sphère creuse élastoplastique avec radoucissement, Journal de Mécanique Appliquée, vol. 1, n¹, 1977, pp. 35-109. NGUYEN M. D., BEREST P., (1979) - Etude de la stabilité des cavités souterraines avec un modèle de comportement

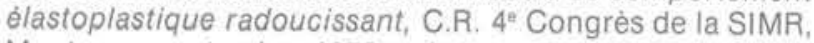
Montreux, septembre 1979, vol. 1, pp. 249-256.

PANET M., (1976), - Analyse de la stabilité d'un tunnel creusé dans un massif rocheux en tenant compte du comportement après la rupture, Rock Mechanics, vol. 8, n॰4. novembre 1976, pp. 209-223.

TINCELIN E., (1979) - La convergence et le soutẻnement provisoire, Journée d'Etudes de l'AFTES, 26 octobre 1978 , Tunnels et Ouvrages Souterrains, $n^{\circ} 32$, avril 1979, pp. 117 119. 